\title{
Performance of the Product of Three Nakagami-m Random Variables
}

\author{
Dragana S. Krstic, Petar B. Nikolic, Ivan Vulic, Sinisa Minic, and Mihajlo C. Stefanovic
}

\begin{abstract}
An output signal from a multi-section wireless relay communication system is equal to the product of the signal envelopes from individual sections. In this paper, a three-sections relay system is considered in the presence of Nakagami- $m$ fading at each section. First, random variable (RV) is formed as the product of three Nakagami-m RVs. For such product, the moments are determined in the closed forms. The first moment is the mean of the signal; the second moment is the average power of the signal, and the third moment is skewness. Then, the Amount of Fading (AoF) is calculated. AoF is a measure of the severity effect of fading in a particular channel model. Besides, all system performance are shown graphically and the parameters influence has been analyzed and discussed.
\end{abstract}

Index Terms-Amount of Fading, Moments, Nakagami-m distribution, Random Variables.

\section{INTRODUCTION}

$\mathrm{S}$ MART city concept start to developed before more than a decade [1]. Smart cities use different types of electronic sensors, Internet of Things (IoT), to collect data, and Internet for their further distribution. The data are collected from citizens and city devices, and then processed and analyzed for better monitoring and manage of city systems [2]-[5]. Due to the multitude of services that need to be provided on a platform that enables Smart City functionality, the question is: which transmission medium can perform better, in terms of price, performance, installation methods, implementation time, etc. So, the question is whether it is better use optical fibers or a wireless way of transmitting data

Manuscript received January 5, 2020; revised April 5, 2020. Date of publication May 27, 2020. Date of current version May 27, 2020.

The part of this paper was presented at the International Conference on Smart and Sustainable Technologies (SpliTech) 2019.

D. S. Krstic and M. C. Stefanovic are with the Faculty of Electronic Engineering, University of Niš, Serbia (e-mails: dragana.krstic@elfak.ni.ac.rs, mihajlo.stefanovic@elfak.ni.ac.rs).

P. Nikolic is with the Tigar Tyres, Pirot, Serbia (nikpetar@gmail.com)

I. Vulic is with the University of Defense, Belgrade, Serbia (e-mail: ivan.vulic@mod.gov.rs).

S. Minic is with Teachers' Training Faculty, Prizren- Leposavic, University of Pristina, Kosovska Mitrovica, Serbia (e-mail: sinisa.minic@pr.ac.rs)

This paper is partially supported by projects: III-44006 and TR-33035 of the Serbian Ministry of Education, Science and Technological Development

Digital Object Identifier (DOI): 10.24138/jcomss.v16i2.989 and information. With the development and improvement of wireless equipment and comprehensive mathematical modeling of wireless channels, the disadvantage in terms of the performance is significantly reduced.

Since it is very important to transmit information without noise and interference, to this end, we consider here the impact of them in wireless communication system. Because of the way signals are transmitted in urban areas, it is important to study the impact of fading, which is main nuisance that occurs during data signals transmission through wireless systems [6].

It is known that the short-term fading of the received envelope in wireless channels can be modelled by means of the Rayleigh, Rician, Nakagami- $m$ and other distributions. For example, Rayleigh distribution is suitable to model amplitude in urban areas, Rician distribution is adequate in sub-urban areas where the line of sight (LOS) components exist, then scintillation effects are good described by Hoyt distribution. However, it is proved by many experiments at both, indoor and outdoor radio channel propagation, that the Nakagami- $m$ distribution offers a better matching for a wider range of fading conditions in wireless communications.

Given that Nakagami- $m$ distribution encompasses several distributions as special cases, this is a generalized way to model small scale fading. Means if we have made the wireless system analysis for Nakagami- $m$ fading, it is equally applicable in any of the above mentioned fading environments and also for several others [6]. Namely, for $m=1$ the Rayleigh distribution will be obtained, for $m>1$ this will be Rician distribution, $m=1 / 2$ gives Gaussian distribution, and for $m<1$ the Hoyt distribution will arise. Therefore, Nakagami- $m$ is generic and wide and that is why we work with it here.

Nowadays, the analysis of wireless telecommunication systems performance is substantially important. Significant attention is dedicated to cascaded fading channels which appear in wireless multi-hop transmission. This is an efficient technology for increasing the coverage with respect to the channel path-loss. The received signals are created as the products of a large number of rays reflected via $N$ statistically independent scatters [7]. Therefore, the analysis of statistics of products of two or more random variables (RVs) is intensified because of their applicability in performance analysis of wireless relay communication systems with more hops (sections) [7] - [15]. 
The wireless relay system output' signal is product of each section' output signal envelope.

The system performance of the first- and second-order are studied in many articles. So, the statistical characteristics of the first order: the outage probability $\left(\mathrm{P}_{\text {out }}\right)$, the amount of fading (AoF) and the bit error probability (BEP) are considered in [9]-[13]. The statistical characteristics of the second order: level crossing rate (LCR) and average fade duration (AFD) are evaluated in [14]-[17].

The expressions for probability density function (PDF) and cumulative distribution function (CDF) for a product of RVs with Rayleigh distribution are derived in [8] across the Meijer G-function. For obtaining the PDFs of products of Rayleigh and Nakagami- $m$ RVs in [9], Mellin transform was utilized.

In [10] an analytical model for evaluation the performance of dual-hop amplify-and-forward (AF) relay transmission over Ricean fading channels on both sections (hops). The PDF and moment generating function (MGF) of received signal-tonoise ratio (SNR) are performed in the form of fast converging sum. Next to, appropriate semi-closed form formulae for average bit error rate (BER) and $\mathrm{P}_{\text {out }}$ was obtained.

The two-hops wireless relay communication radio system subjected to $\kappa-\mu$ short-term fading was analyzed in [11] and the $\mathrm{P}_{\text {out }}$ is calculated using sums and modified Bessel functions of the second kind.

The work [12] deals with the product of three arbitrary, independent and non-identically distributed $\alpha-\mu$ RVs. There, the expressions for the PDF, the CDF, and the moments of the resulting distribution are derived in closed-forms. This $\alpha-\mu$ model is general fading model since includes a big number of fading distributions for different values of parameters $\alpha$ and $\mu$.

The second order statistical characteristics of the AF multihop Rayleigh fading channel are investigated in [13]. Here, the expressions for the average LCR and AFD of the product of $N$ Rayleigh fading amplitudes are obtained. A match for the previously published case of product of two Rayleigh RVs has been proven.

The expressions and closed-form approximations for the LCR and the AFD of the double Nakagami- $m$ random variable are calculated in [14] and utilized for analysis of the second order characteristics of multiple input multiple output (MIMO) keyhole fading environment.

The product of $N$ statistically independent RVs is known as $N *$ Nakagami RV. It is suitable for presenting cascaded Nakagami- $m$ channels. Its distribution is given in [15] and the MGF, PDF, CDF, and moments are derived in closed forms using the Meijer's $G$ function. Afterwards the expressions for the OP, AoF, and average BEP for different modulations in the presence of $N *$ Nakagami fading are obtained in closed-forms.

In [18], the PDF and CDF, of the wireless relay communication system with three sections in the presence of Nakagami- $m$ fading are determined. The LCR of product of three Nakagami- $m$ RVs is presented in simple closed-form expression in [19]. The LCR is a criterion of the rapidity of the fading which displays how often the fading crosses defined threshold, usually in the positive-going direction. Further, the AFD of the wireless relay system with three sections under the presence of Nakagami- $m$ fading is obtained in [20]. AFD is used to show how long a user is in continuous outage, below a set specified threshold level, after crossing this level in a downward direction. In this work, for such system we will derive moments and Amount of fading.

The remainder of this article is organized as follows. In the Section II, all system performance of the first order of product of three Nakagami- $m$ RVs are listed or derived. Section III presents the second order performance of triple Nakagami- $m$ RV with some plots of the obtained performance metrics. Finally, Section IV gives the final comments and indications about future investigation.

\section{THE FIRST ORDER PERFORMANCE OF PRODUCT OF THREE NAKAGAMI-M RANDOM VARIABLES}

Random variables $\quad x_{i}, \quad i=1,2,3$ have Nakagami- $m$ distributions [21]:

$$
p_{x_{i}}\left(x_{i}\right)=\frac{2}{\Gamma\left(m_{i}\right)}\left(\frac{m_{i}}{\Omega_{i}}\right)^{m_{i}} x_{i}^{2 m_{i}-1} e^{-\frac{m_{i}}{\Omega_{i}} x_{i}^{2}}, x_{i} \geq 0
$$

where $\Gamma($.$) is the gamma function, \Omega_{i}$ are powers of RVs $x_{i}$ and $m_{i}$ are fading severity parameters of Nakagami- $m$ RVs $x_{i}$. The value of the shape parameter $m$ indicates the severity of multipath fading. It is a measure of channel quality and is necessary for many applications to evaluate it.

Let suppose random variable (RV) $x$ is defined as the product of three Nakagami- $m$ RVs:

$$
x=x_{1} x_{2} x_{3}=\prod_{i=1}^{3} x_{i} .
$$

\section{A. PDF of Product of Three Nakagami-m RVs}

The PDF of product of three Nakagami- $m$ RVs $x$ is given by Eq. (13) in [18]:

$$
\begin{gathered}
p_{x}(x)=\frac{8}{\Gamma\left(m_{1}\right) \Gamma\left(m_{2}\right) \Gamma\left(m_{3}\right)}\left(\frac{m_{1}}{\Omega_{1}}\right)^{m_{1}}\left(\frac{m_{2}}{\Omega_{2}}\right)^{m_{2}}\left(\frac{m_{3}}{\Omega_{3}}\right)^{m_{3}} . \\
x^{2 m_{3}-1} \cdot\left(\frac{m_{1} \Omega_{3}}{m_{3} \Omega_{1}}\right)^{m_{3}-m_{1}} \\
\cdot \int_{0}^{\infty} d x_{2} x_{2}^{2 m_{2}-2 m_{3}-1} e^{\frac{m_{2}}{\Omega_{2}} x_{2}^{2}} \cdot K_{2 m_{3}-2 m_{1}}\left(2 \sqrt{\frac{m_{1} m_{3}}{\Omega_{1} \Omega_{3}} \frac{x^{2}}{x_{2}^{2}}}\right)
\end{gathered}
$$

where $K_{n}(x)$ denotes the modified Bessel function of the second kind [22, eq. (3.471.9)].

The curves for PDF of product of three Nakagami- $m$ RVs are shown in Fig. 1 in [20] depending on this product $x$ for some values of distribution parameters: Nakagami- $m$ fading severity $m_{i}$ and signal average powers $\Omega_{i}$ at sections.

It is visible that for all values of Nakagami- $m$ severity parameters $m_{i}$ and signal powers $\Omega_{i}$ PDF increases at low values of $x$, achieves maximum and then decrease for bigger values of the signal envelope. The low values of $\mathrm{x}$ affect more on the size of the PDF. 


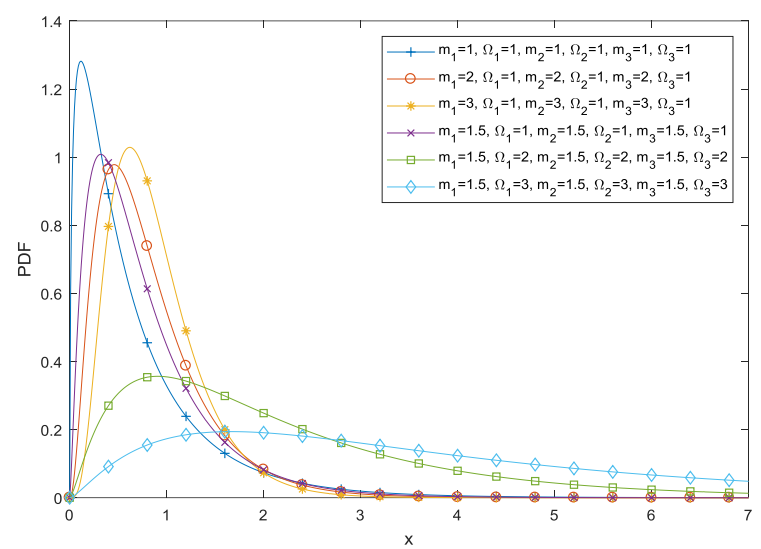

Fig. 1. PDF of product of three Nakagami- $m$ RVs for different values of Nakagami- $m$ fading severity parameters $m_{l}$ and signal power $\Omega_{1}$.

\section{B. CDF of Product of Three Nakagami-m RVs}

The CDF of product of three Nakagami- $m$ RVs $x$ has been derived in [[18], Eq. (16)]:

$$
\begin{gathered}
F_{x}(x)=\int_{0}^{x} d t p_{x}(t)= \\
=\frac{8}{\Gamma\left(m_{1}\right) \Gamma\left(m_{2}\right) \Gamma\left(m_{3}\right)}\left(\frac{m_{1}}{\Omega_{1}}\right)^{m_{1}}\left(\frac{m_{2}}{\Omega_{2}}\right)^{m_{2}}\left(\frac{m_{3}}{\Omega_{3}}\right)^{m_{3}} . \\
\cdot \frac{1}{m_{1}} x^{2 m_{1}} \cdot \sum_{j_{1}=0}^{\infty} \frac{1}{\left(m_{1}+1\right)\left(j_{1}\right)} \frac{m_{1}^{j_{1}} x^{2 j_{1}}}{\Omega_{1}^{j_{1}}} . \\
\cdot \int_{0}^{\infty} d x_{2} x_{2}^{2 m_{2}-2 m_{3}-1} e^{-\frac{m_{2}}{\Omega_{2}} x_{2}^{2}} \cdot \\
\cdot\left(\frac{m_{1} \Omega_{3}}{m_{3} \Omega_{1}} x^{2}\right)^{m_{3}-m_{1}-j_{1}} \cdot K_{2 m_{3}-2 m_{1}-2 j_{1}}\left(2 \sqrt{\frac{m_{1} m_{3} x^{2}}{\Omega_{1} \Omega_{2} x_{2}^{2}}}\right) .
\end{gathered}
$$

\section{C. $P_{\text {out }}$ of Product of Three Nakagami-m RVs}

The outage probability, $P_{\text {out }}$, is defined as probability that the receiver output signal envelope, or signal to interference ratio (SNR), falls below a given threshold value. $P_{\text {out }}$ is therefore defined as [[23], eq. (2.23)]:

$$
P_{\text {out }}=P(X \leq x)=\int_{0}^{x} d t p_{x}(t),
$$

whereby $x$ is this given threshold value.

Mathematically, $P_{\text {out }}$ is actually the CDF of the signal:

$$
P_{\text {out }}=F_{x}(x),
$$

and it can be expressed as Eq. (4).

Graphs of the outage probability are presented in Fig. 2 in [20] for a few different values of the parameters. The choice of parameters can illustrate the range of shapes that the curves of the resulting distribution can have.

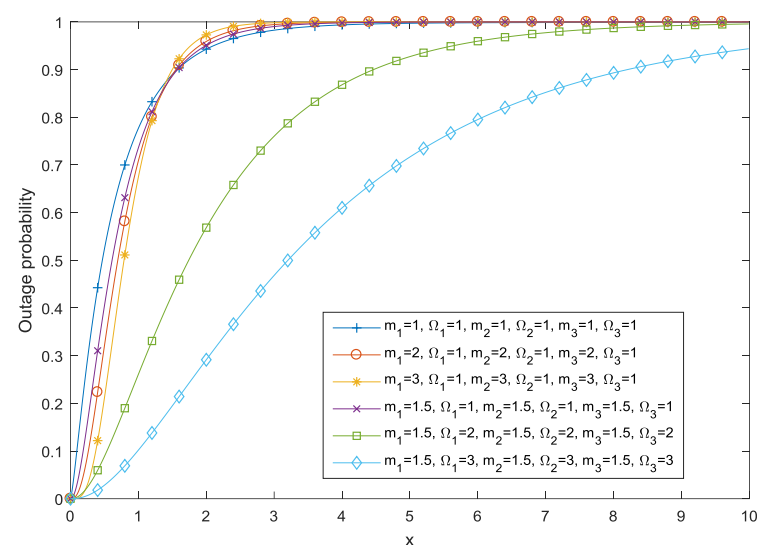

Fig. 2. The outage probability of product of three Nakagami- $m$ RVs for different values of Nakagami- $m$ fading severity parameters $m_{l}$ and signal power $\Omega_{1}$.

One can recognize from the figure that $P_{\text {out }}$ is increasing for all values of the range of the signal envelope and fast achieves saturation. At low $x$, the $P_{\text {out }}$ shrinks as the omega grows and severity parameters $m_{i}$ increase, as it was expected.

\section{Moments of Product of Three Nakagami-m RVs}

In mathematics, moment is quantitative measure of the shape of function. It is used in statistics and mechanics also. If this function is probability distribution, then the zero-th moment is the total probability, the first moment is the expected value or mean of the signal, the second moment is the variance or the average power of signal, the third moment is the skewness, and the fourth moment is the kurtosis.

The mean of the signal, or the first moment $m_{l}$, is defined as:

$$
m_{1}=\bar{x}=\int_{0}^{\infty} d x x p_{x}(x)
$$

In the continuation of this paper we will perform the closedform formula for the first moment of this product of three Nakagami- $m$ RVs.

$$
\begin{aligned}
& m_{1}=\frac{8}{\Gamma\left(m_{1}\right) \Gamma\left(m_{2}\right) \Gamma\left(m_{3}\right)}\left(\frac{m_{1}}{\Omega_{1}}\right)^{m_{1}}\left(\frac{m_{2}}{\Omega_{2}}\right)^{m_{2}}\left(\frac{m_{3}}{\Omega_{3}}\right)^{m_{3}} . \\
& \int_{0}^{\infty} d x_{2} x_{2}^{-2 m_{1}+2 m_{2}-1} e^{-\frac{m_{2}}{\Omega_{2}} x_{2}^{2}} \int_{0}^{\infty} d x_{3} x_{3}^{-2 m_{1}+2 m_{3}-1} e^{-\frac{m_{3}}{\Omega_{3}} x_{3}^{2}} . \\
& \int_{0}^{\infty} d x x^{1+2 m_{1}-1} e^{-\frac{m_{1}}{\Omega_{1}} \frac{x^{2}}{x_{2}^{2} x_{3}^{2}}}= \\
& =\frac{8}{\Gamma\left(m_{1}\right) \Gamma\left(m_{2}\right) \Gamma\left(m_{3}\right)}\left(\frac{m_{1}}{\Omega_{1}}\right)^{m_{1}}\left(\frac{m_{2}}{\Omega_{2}}\right)^{m_{2}}\left(\frac{m_{3}}{\Omega_{3}}\right)^{m_{3}} . \\
& \int_{0}^{\infty} d x_{2} x_{2}^{-2 m_{1}+2 m_{2}-1} e^{-\frac{m_{2}}{\Omega_{2}} x_{2}^{2}} \int_{0}^{\infty} d x_{3} x_{3}^{-2 m_{1}+2 m_{3}-1} e^{-\frac{m_{3}}{\Omega_{3}} x_{3}^{2}} .
\end{aligned}
$$




$$
\begin{aligned}
& \frac{1}{2}\left(\frac{\Omega_{1}}{m_{1}}\right)^{m_{1}+1 / 2} \Gamma\left(m_{1}+1 / 2\right)\left(x_{2}^{2} x_{3}^{2}\right)^{m_{1}+1 / 2}= \\
& =\frac{8}{\Gamma\left(m_{1}\right) \Gamma\left(m_{2}\right) \Gamma\left(m_{3}\right)}\left(\frac{m_{1}}{\Omega_{1}}\right)^{m_{1}}\left(\frac{m_{2}}{\Omega_{2}}\right)^{m_{2}}\left(\frac{m_{3}}{\Omega_{3}}\right)^{m_{3}} . \\
& \frac{1}{2}\left(\frac{\Omega_{1}}{m_{1}}\right)^{m_{1}+1 / 2} \Gamma\left(m_{1}+1 / 2\right) . \\
& \int_{0}^{\infty} d x_{2} x_{2}^{-2 m_{1}+2 m_{2}-1+2 m_{1}+1} e^{-\frac{m_{2}}{\Omega_{2}} x_{2}^{2}} . \\
& \int_{0}^{\infty} d x_{3} x_{3}^{-2 m_{1}+2 m_{3}-1+2 m_{1}+1} e^{-\frac{m_{3}}{\Omega_{3}} x_{3}^{2}}= \\
& =\frac{8}{\Gamma\left(m_{1}\right) \Gamma\left(m_{2}\right) \Gamma\left(m_{3}\right)}\left(\frac{m_{1}}{\Omega_{1}}\right)^{m_{1}}\left(\frac{m_{2}}{\Omega_{2}}\right)^{m_{2}}\left(\frac{m_{3}}{\Omega_{3}}\right)^{m_{3}} . \\
& \cdot \frac{1}{2}\left(\frac{\Omega_{1}}{m_{1}}\right)^{m_{1}+1 / 2} \Gamma\left(m_{1}+1 / 2\right) \frac{1}{2}\left(\frac{\Omega_{2}}{m_{2}}\right)^{m_{2}+1 / 2} \Gamma\left(m_{2}+1 / 2\right) \\
& \frac{1}{2}\left(\frac{\Omega_{3}}{m_{3}}\right)^{m_{3}+1 / 2} \Gamma\left(m_{3}+1 / 2\right)= \\
& =\frac{1}{\Gamma\left(m_{1}\right) \Gamma\left(m_{2}\right) \Gamma\left(m_{3}\right)}\left(\frac{\Omega_{1}}{m_{1}}\right)^{1 / 2}\left(\frac{\Omega_{2}}{m_{2}}\right)^{1 / 2}\left(\frac{\Omega_{3}}{m_{3}}\right)^{1 / 2} . \\
& \Gamma\left(m_{1}+1 / 2\right) \Gamma\left(m_{2}+1 / 2\right) \Gamma\left(m_{3}+1 / 2\right) .
\end{aligned}
$$

In Fig. 3, the first moment for product of three Nakagami- $m$ RVs versus Nakagami- $m$ fading severity parameter $m\left(m=m_{l}=\right.$ $\left.m_{2}=m_{3}\right)$, for some values of the signal power $\Omega$ $\left(\Omega=\Omega_{1}=\Omega_{2}=\Omega_{3}\right)$ is drawn.

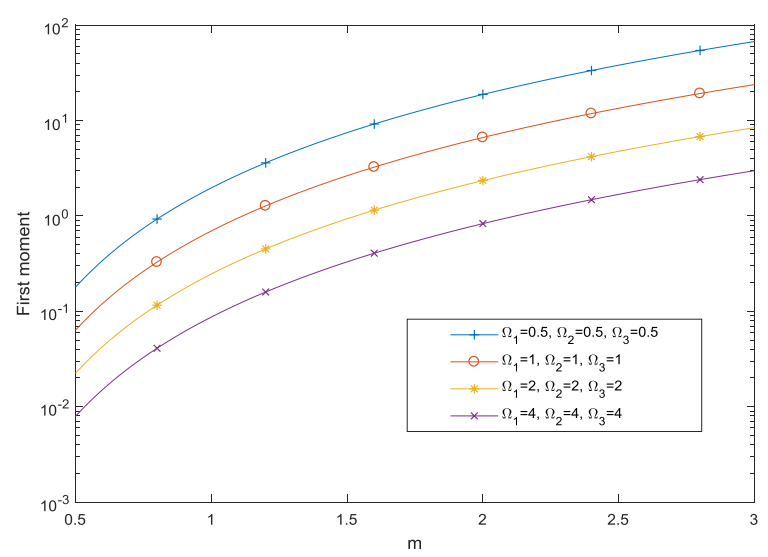

Fig. 3. The first moment for product of three Nakagami- $m$ RVs depending on Nakagami- $m$ fading severity parameters $\mathrm{m}_{\mathrm{i}}$ for a few values of signal power $\Omega=\Omega_{1}=\Omega_{2}=\Omega_{3}$

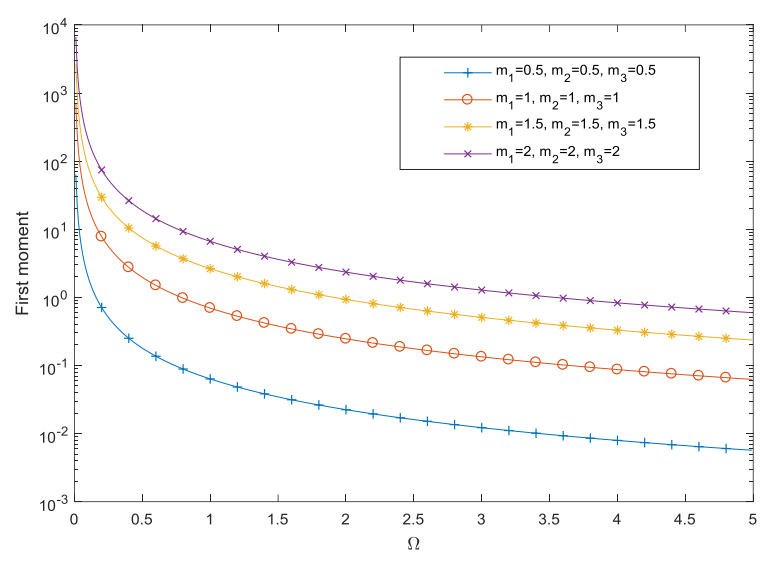

Fig. 4. The first moment for product of three Nakagami- $m$ RVs versus signal power $\Omega=\Omega_{1}=\Omega_{2}=\Omega_{3}$ for different Nakagami- $m$ severity parameters $m_{i}$

It is possible to catch sight of increasing the first moment with increasing of Nakagami-m fading severity parameter $\mathrm{m}$. Also, the first moment, or mean of signal, increases with decreasing of the signal power $\Omega$ i.

The first moment of the product of three Nakagami-m RVs depending on the signal power is presented in Fig. 4. Here, the parameter of curves is Nakagami-m fading severity parameter mi. One can discern from this figure that the first moment of product of three Nakagami-m RVs declines with the rise of $\Omega$, and it is confirmed that decreases for larger mi.

The second moment, $m_{2}$, is defined by:

$$
m_{2}=\overline{x^{2}}=\int_{0}^{\infty} d x x^{2} p_{x}(x) .
$$

Now comes the derivation of the second moment of the product of three Nakagami- $m$ RVs.

$$
\begin{aligned}
& m_{2}=\frac{8}{\Gamma\left(m_{1}\right) \Gamma\left(m_{2}\right) \Gamma\left(m_{3}\right)}\left(\frac{m_{1}}{\Omega_{1}}\right)^{m_{1}}\left(\frac{m_{2}}{\Omega_{2}}\right)^{m_{2}}\left(\frac{m_{3}}{\Omega_{3}}\right)^{m_{3}} . \\
& \int_{0}^{\infty} d x_{2} x_{2}^{-2 m_{1}+2 m_{2}-1} e^{-\frac{m_{2}}{\Omega_{2}} x_{2}^{2}} \int_{0}^{\infty} d x_{3} x_{3}^{-2 m_{1}+2 m_{3}-1} e^{-\frac{m_{3}}{\Omega_{3}} x_{3}^{2}} . \\
& \int_{0}^{\infty} d x x^{2+2 m_{1}-1} e^{-\frac{m_{1}}{\Omega_{1}} x_{2}^{2} x_{3}^{2}}= \\
& \int_{0}^{\infty} d x_{2} x_{2}^{-2 m_{1}+2 m_{2}-1} e^{-\frac{m_{2}}{\Omega_{2}} x_{2}^{2}} \int_{0}^{\infty} d x_{3} x_{3}^{-2 m_{1}+2 m_{3}-1} e^{-\frac{m_{3}}{\Omega_{3}} x_{3}^{2}} . \\
& \left.\frac{1}{2}\left(\frac{\left.m_{1}\right) \Gamma\left(m_{3}\right)}{m_{1}}\right)^{m_{1}+1}\right)^{\Gamma\left(m_{1}+1\right)\left(x_{2}^{2} x_{3}^{2}\right)^{m_{1}+1}}=
\end{aligned}
$$




$$
\begin{gathered}
=\frac{4}{\Gamma\left(m_{1}\right) \Gamma\left(m_{2}\right) \Gamma\left(m_{3}\right)}\left(\frac{m_{1}}{\Omega_{1}}\right)^{m_{1}}\left(\frac{m_{2}}{\Omega_{2}}\right)^{m_{2}}\left(\frac{m_{3}}{\Omega_{3}}\right)^{m_{3}} . \\
\cdot\left(\frac{\Omega_{1}}{m_{1}}\right)^{m_{1}+1} \Gamma\left(m_{1}+1\right) \cdot \\
\cdot \frac{1}{2}\left(\frac{\Omega_{2}}{m_{2}}\right)^{m_{2}+1} \Gamma\left(m_{2}+1\right) \cdot \frac{1}{2}\left(\frac{\Omega_{3}}{m_{3}}\right)^{m_{3}+1} \Gamma\left(m_{3}+1\right)= \\
=\frac{1}{\Gamma\left(m_{1}\right) \Gamma\left(m_{2}\right) \Gamma\left(m_{3}\right)}\left(\frac{\Omega_{1}}{m_{1}}\right)\left(\frac{\Omega_{2}}{m_{2}}\right)\left(\frac{\Omega_{3}}{m_{3}}\right) . \\
\Gamma\left(m_{1}+1\right) \Gamma\left(m_{2}+1\right) \Gamma\left(m_{3}+1\right) .
\end{gathered}
$$

The second moment is actually the variance. The positive square root of the variance is the standard deviation. In wireless communication this is the average power of the signal.

The influence of parameters of fading distribution to the second moment of product of three Nakagami- $m$ RVs can be noticed from the next two figures.

One can see from Figs 5. and 6. that the second moment, or variance, increases with increasing of Nakagami- $m$ fading severity parameter $m$ and increases with reduction of the signal power $\Omega_{\mathrm{i}}$.

The normalized third central moment is called the skewness. In probabilistic theory and statistics, the skewness is a measure of the limitedness of the distribution or the asymmetry of the probability distribution of a real-valued random variable $(\mathrm{RV})$ about its mean.

The skewness value can be positive or negative, or even undefined. The symmetric distributions have the third moment equal to zero, if it is defined. The distribution that is skewed to the left (it means that the tail of the distribution is longer on the left side of the curve) has negative skewness and the distribution which is skewed to the right has positive skewness.

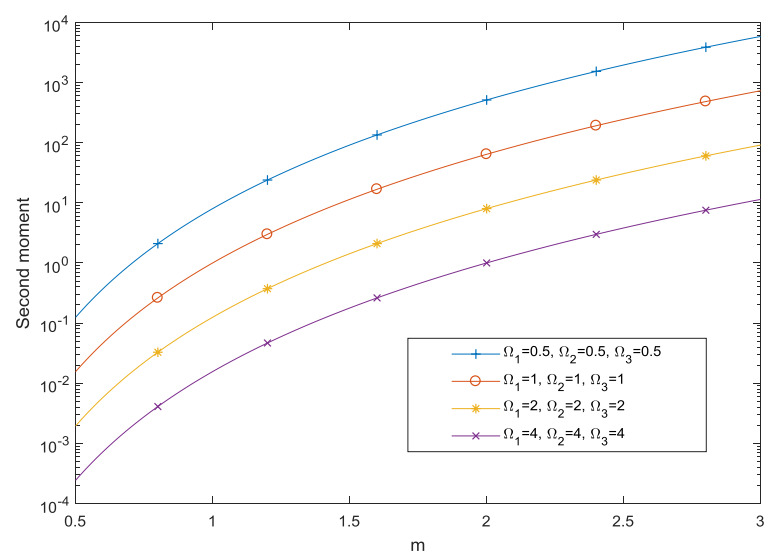

Fig. 5. The second moment of product of three Nakagami- $m$ RVs versus Nakagami- $m$ fading severity parameter $m=m_{l}=m_{2}=m_{3}$ for different values of signal power $\Omega_{\mathrm{i}}$.

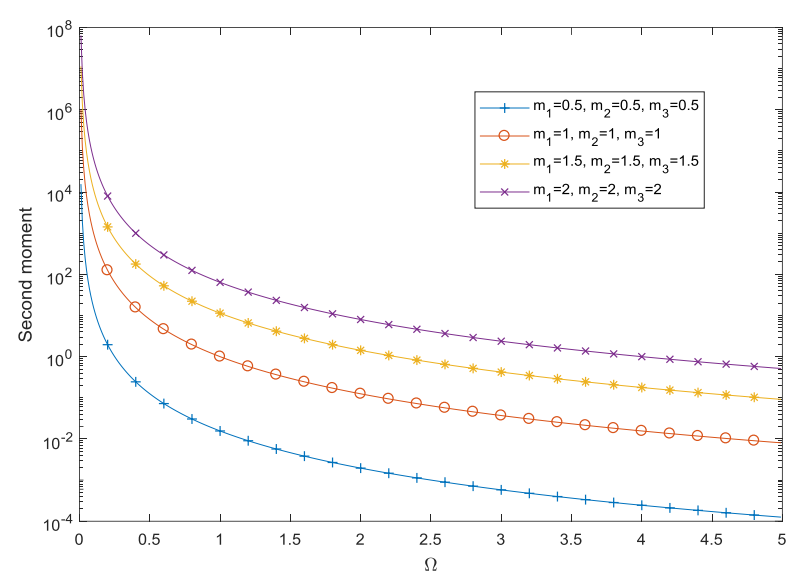

Fig. 6. The second moment of product of three Nakagami- $m$ RVs depending on signal power $\Omega=\Omega_{1}=\Omega_{2}=\Omega_{3}$ for different values of Nakagami- $m$ fading severity parameters $m_{i}$.

The third moment, $m_{3}$ is given as:

$$
m_{3}=\overline{x^{3}}=\int_{0}^{\infty} d x x^{3} p_{x}(x)
$$

The final formula is derived in the similar way as previous two moments:

$$
\begin{gathered}
m_{3}=\frac{1}{\Gamma\left(m_{1}\right) \Gamma\left(m_{2}\right) \Gamma\left(m_{3}\right)}\left(\frac{\Omega_{1}}{m_{1}}\right)^{3 / 2}\left(\frac{\Omega_{2}}{m_{2}}\right)^{3 / 2}\left(\frac{\Omega_{3}}{m_{3}}\right)^{3 / 2} . \\
\cdot \Gamma\left(m_{1}+3 / 2\right) \cdot \Gamma\left(m_{2}+3 / 2\right) \cdot \Gamma\left(m_{3}+3 / 2\right) .
\end{gathered}
$$

Graphs of the dependence of the third moment on Nakagami- $m$ fading severity parameter $m$ and signal power $\Omega$ are drawn in Figs. 7 and 8 respectively.

It is clear from the first plot that higher values of the Nakagami-m fading severity parameter $m$ tends to increase the third moment. On the other hand, with the rise of the signal power $\Omega$, third moment becomes smaller, as expected.

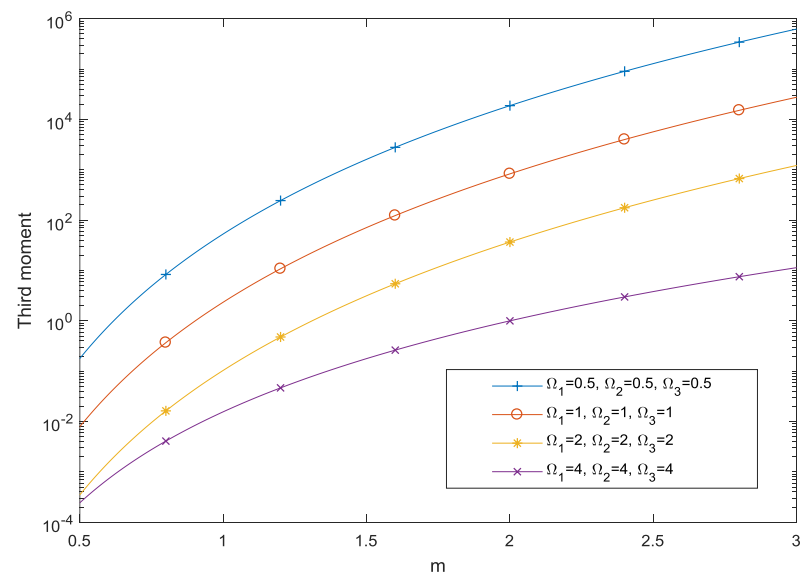

Fig. 7. The third moment of product of three Nakagami- $m$ RVs versus Nakagami- $m$ severity parameters $m_{i}$ for different values of signal power $\Omega_{1}$. 


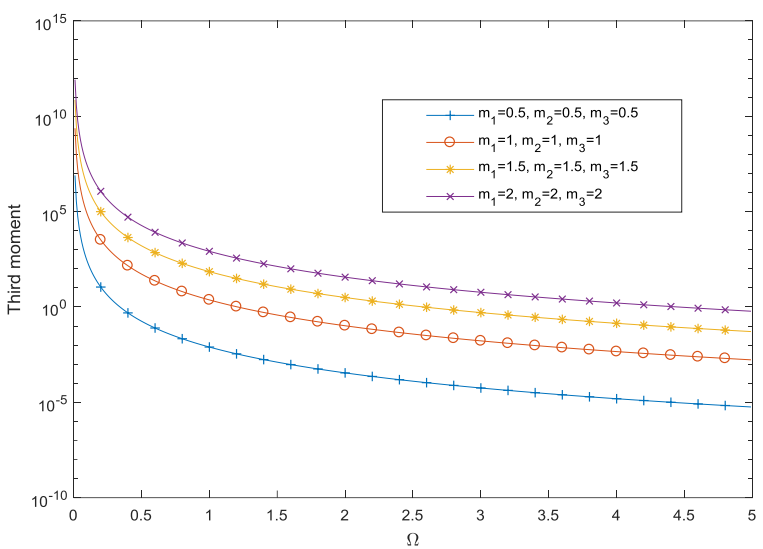

Fig. 8. The third moment of product of three Nakagami- $m$ RVs depending on signal power $\Omega=\Omega_{1}=\Omega_{2}=\Omega_{3}$ for different values of Nakagami- $m$ fading severity parameters $m_{i}$

In the theory of probability and statistics, the fourth moment, the kurtosis, is a measure of the "tailness" of the PDF of a RV. Like skewness, kurtosis describes the shape of a probability distribution. Pearson defined in [24] kurtosis to measure departure from normality, and gave the terms "leptokurtic," "mesokurtic," and "platykurtic" to indicate where kurtosis is bigger than 0 , equal to 0 , and less than 0 .

As previous moments, the kurtosis is derived in the shape:

$$
\begin{gathered}
m_{4}=\frac{1}{\Gamma\left(m_{1}\right) \Gamma\left(m_{2}\right) \Gamma\left(m_{3}\right)}\left(\frac{\Omega_{1}}{m_{1}}\right)^{2}\left(\frac{\Omega_{2}}{m_{2}}\right)^{2}\left(\frac{\Omega_{3}}{m_{3}}\right)^{2} . \\
\cdot \Gamma\left(m_{1}+2\right) \cdot \Gamma\left(m_{2}+2\right) \cdot \Gamma\left(m_{3}+2\right) .
\end{gathered}
$$

Finally, the $n$-th moment, $m_{n}$, of the product of three Nakagami- $m$ RVs is:

$$
\begin{gathered}
m_{n}=\overline{x^{n}}=\int_{0}^{\infty} d x x^{n} p_{x}(x)= \\
=\frac{1}{\Gamma\left(m_{1}\right) \Gamma\left(m_{2}\right) \Gamma\left(m_{3}\right)}\left(\frac{\Omega_{1}}{m_{1}}\right)^{n / 2}\left(\frac{\Omega_{2}}{m_{2}}\right)^{n / 2}\left(\frac{\Omega_{3}}{m_{3}}\right)^{n / 2} . \\
\Gamma\left(m_{1}+n / 2\right) \Gamma\left(m_{2}+n / 2\right) \Gamma\left(m_{3}+n / 2\right) .
\end{gathered}
$$

\section{E. AoF of Product of Three Nakagami-m RVs}

The amount of fading is a simple measure for the severity of fading for given channel. To obtain this performance, the moments of the fading distribution itself are directly used. Thus, it is a simple but effective way to quantify fading.

AoF is independent of the mean fading power, but depends only on the current signal, or SNR. It is specified by means of the equation [25, Eq. (9)]:

$$
A o F=\frac{E\left[x^{2}\right]}{\{E[x]\}^{2}}-1 .
$$

In Fig. 9, Amount of fading of product of three Nakagami$m$ RVs is presented versus Nakagami- $m$ fading severity parameters $m$ for more $\Omega$.

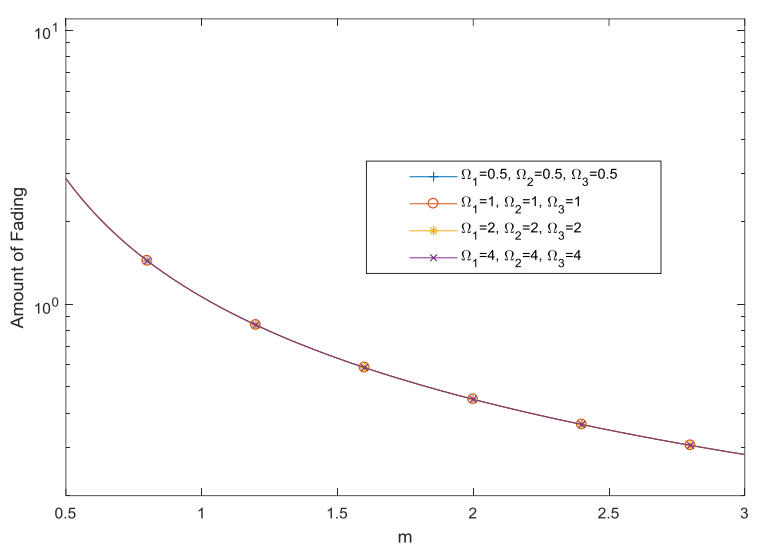

Fig. 9. The Amount of fading of product of three Nakagami- $m$ RVs depending on the Nakagami- $m$ fading severity parameters $m_{i}$ for different signal powers $\Omega_{\mathrm{i}}$.

Hence, AoF can be obtained directly from the moments as:

$$
A o F=\frac{m_{2}}{m_{1}^{2}}-1
$$

It is visible from this graph that $\mathrm{AoF}$ decreases with increasing of $m$. Also, it can be seen that AoF does not depend of $\Omega$. This theoretical assumption of Aof's independence on the signal power is confirmed by Fig. 10 .

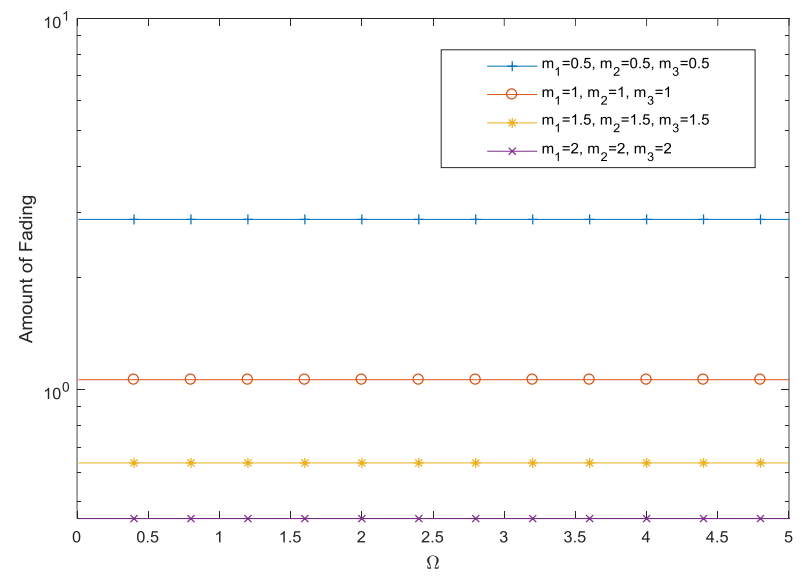

Fig. 10. The amount of fading of product of three Nakagami- $m$ RVs versus signal power $\Omega$ for some values of Nakagami- $m$ fading severity parameters $m_{i}$

\section{The SECONd ORder PERFormance OF THE PRODUCT OF THREE NAKAGAMI-M RANDOM VARIABLES}

The level crossing rate and the average fade duration are second order characteristics, very important for better understanding of the behavior of the wireless channels.

\section{A. LCR of Product of Three Nakagami-m RVs}

The LCR of $X$ at threshold $x$ is defined as the rate at which the random process crosses a given level $x$ in the negative (or positive) direction [26, Eq. (2.106)]. To derive the LCR, a knowledge of the joint probability density function (JPDF) between $x$ and $\dot{x}, p_{x \dot{x}}(x \dot{x})$, and application of the Rice's formula [27, Eq. (2.106)] is required: 


$$
N_{X}(x)=\int_{0}^{\infty} d \dot{x} \dot{x} p_{x \dot{x}}(x \dot{x}) .
$$

The LCR of product $x$ of three Nakagami- $m$ RVs is obtained in [19, Eq. (18)] after necessary replacements in upper formula:

$$
\begin{gathered}
N_{X}=\int_{0}^{\infty} d x_{2} \int_{0}^{\infty} d x_{3}\left(\int_{0}^{\infty} d \dot{x} \dot{x} p_{\dot{x}}\left(\dot{x} / x x_{2} x_{3}\right) \frac{1}{x_{2} x_{3}} p_{x_{1}}\left(\frac{x}{x_{2} x_{3}}\right) p_{x_{2}}\left(x_{2}\right) p_{x_{3}}\left(x_{3}\right)\right)= \\
=\int_{0}^{\infty} d x_{2} \int_{0}^{\infty} d x_{3} \frac{1}{\sqrt{2 \pi}} \sigma_{\dot{x}} \frac{1}{x_{2} x_{3}} p_{x_{1}}\left(\frac{x}{x_{2} x_{3}}\right) p_{x_{2}}\left(x_{2}\right) p_{x_{3}}\left(x_{3}\right)= \\
=\frac{1}{\sqrt{2 \pi}} \pi f_{m} \frac{\Omega_{1}^{1 / 2}}{m_{1}^{1 / 2}} x^{2 m_{1}-1} \frac{2}{\Gamma\left(m_{1}\right)} \frac{2}{\Gamma\left(m_{2}\right)} \frac{2}{\Gamma\left(m_{3}\right)} \cdot \\
\cdot\left(\frac{m_{1}}{\Omega_{1}}\right)^{m_{1}}\left(\frac{m_{2}}{\Omega_{2}}\right)^{m_{2}}\left(\frac{m_{3}}{\Omega_{3}}\right)^{m_{1}} \cdot \\
\cdot \int_{0}^{\infty} d x_{2} \int_{0}^{\infty} d x_{3} x_{2}^{-2 m_{1}+2 m_{2}} x_{3}^{-2 m_{1}+2 m_{3}} e^{-\frac{m_{1}}{\Omega_{1}} x_{2}^{2} x_{3}^{2}-\frac{m_{2}}{\Omega_{2}} x_{2}^{2}-\frac{m_{3}}{\Omega_{3}} x_{3}^{2}} \\
\cdot\left(1+\frac{x^{2}}{x_{2}^{4} x_{3}^{2}} \frac{\Omega_{2}}{\Omega_{1}} \frac{m_{1}}{m_{2}}+\frac{x^{2}}{x_{2}^{2} x_{3}^{4}} \frac{\Omega_{3}}{\Omega_{1}} \frac{m_{1}}{m_{3}}\right)^{1 / 2},
\end{gathered}
$$

and solved by using Laplace approximation theorem [28].

In Fig. 11 is shown Fig. 2 from [19], the dependence of the LCR normalized by $f_{m}$ on signal envelope for different values of Nakagami- $m$ fading severity parameters $m_{2}$ and signal power $\Omega_{2}$. One can conclude that LCR increases for low values of the signal envelope, reaches out the maximum and start to decline for higher values of $x$. LCR is more affected by small values of the signal envelope.

Better system performance are achaived with smaller values of the LCR.

\section{B. AFD of Product of Three Nakagami-m RVs}

The AFD of $X$ at threshold $x$ is defined as the average time that the product of three Nakagami- $m$ RVs stays below level $x$ after crossing that level in the downward direction.

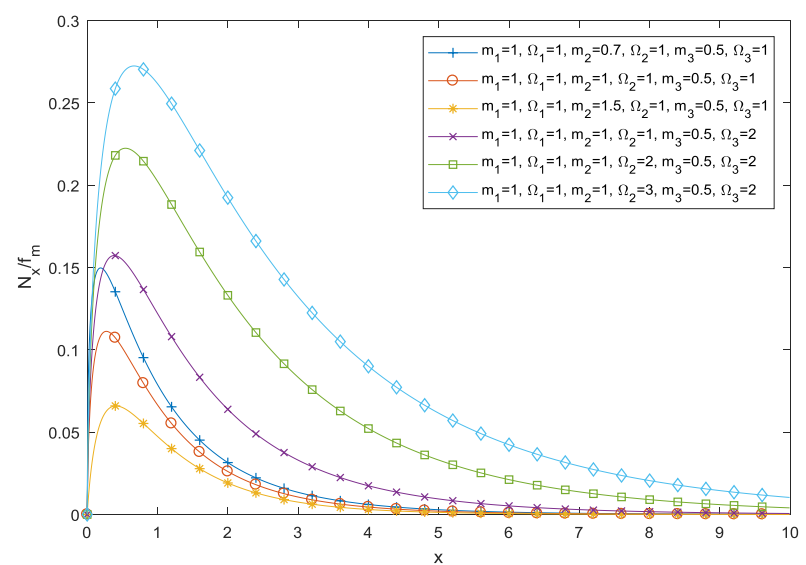

Fig. 11. LCR normalized by $\mathrm{f}_{\mathrm{m}}$ versus signal envelope $x$ for various values of Nakagami- $m$ fading severity parameter $m_{2}$ and signal power $\Omega_{2}$

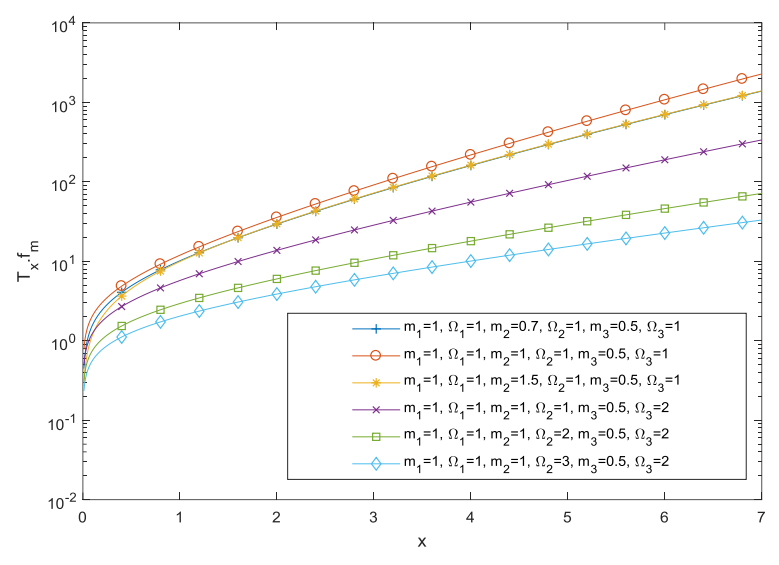

Fig. 12. AFD normalized by $\mathrm{f}_{\mathrm{m}}$ versus signal envelope $x$ for different values of Nakagami- $m$ fading severity parameters $m_{i}$ and signal powers $\Omega_{\mathrm{i}}$.

According to that, AFD is:

$$
T_{x}(x)=\frac{F_{x}(x)}{N_{x}(x)} .
$$

Here $F_{x}(x)$ is the CDF of $x$ from (4), and $N_{x}(x)$ is given in (18).

The normalized AFD $\left(T_{x} f_{m}\right)$ of product of three Nakagami- $m$ RVs was calculated in [20] and plotted in Fig. 12 versus resulting signal envelope $x$. It is visible from this figure that AFD growing for all signal envelopes values and lower $\Omega_{\mathrm{i}}$ and fading severity parameters $m_{i}$. This figure confirms theoretical fact.

\section{CONCLUSION}

In this paper, exact, relatively simple, closed-form expressions for the moments of the product of three Nakagami- $m$ RVs are determined. Based on these results, the amount of fading for the proposed cascaded fading channel is obtained. Earlier, the Pout, LCR and AFD for the same scenario are derived in closed forms and presented here to illustrate all performance of cascaded three-hop Nakagami- $m$ fading channel. As mentioned earlier, because the Nakagami$m$ distribution is general, this result can be used for the cascaded three-hop Rayleigh fading channel if we put Nakagami- $m$ fading severity parameters $m_{i}$ to be equal to 1 . Also, this model is useful for three-hop channel in the presence of Gaussian fading if $m_{i}=1 / 2$. Even if Nakagami- $m$ distribution has advantages versus some other models, yet approximation using Nakagami- $m$ may not be optimal for all cases, especially at tails of the distribution. For these cases, more generalized and accurate models should be used. Such distribution models are: $\alpha-\mu, \kappa-\mu, \eta-\mu, \alpha-\eta-\mu$, etc. Accordingly, our further work can be oriented to multi-hop wireless relay channels with newer general distributions describing fading influence.

\section{REFERENCES}

[1] G. S. Yovanof and G. N. Hazapis, "An architectural framework and enabling wireless technologies for digital cities \& intelligent urban environments," Wireless Personal Communications, 49 (3), March 2009 , pp. 445-463, doi:10.1007/s11277-009-9693-4. 
[2] A. Haidine, S. El Hassani, A. Aqqal, and A. El Hannani, "The role of communication technologies in building future smart cities, 2016, DOI: $10.5772 / 64732$

[3] D. Dobrilović, "Networking technologies for smart cities: an overview," Interdisciplinary Description of Complex Systems 16(3-A), 408-416, 2018. DOI: 10.7906/indecs.16.3.13

[4] I. Yaqoob, I. A. T. Hashem, Y. Mehmood, A. Gani, S. Mokhtar, S Guizani, "Enabling communication technologies for smart cities," IEEE Communications Magazine, 55(1), January 2017, DOI: 10.1109/MCOM /MCOM.2017.1600232CM

[5] T.Q. Duong, N. Vo, , and C. Zhu, "Editorial: Wireless communication and networks for smart cities," Mobile Networks and Applications, vol. 23, 2018, pp. 1522-1524. doi:10.1007/s11036-0181097-9

[6] Andrea Goldsmith, Wireless Communications, Cambridge University Press, 2005.

[7] F. Yilmaz and M. S. Alouini, "Product of the powers of generalized Nakagami- $m$ variates and performance of cascaded fading channels," 2009 IEEE Global Telecommunications Conference, Honolulu, HI, Dec. 2009, pp. 1-8, doi:10.1109/glocom.2009.5426254

[8] J. Salo, H. M. El-Sallabi, and P. Vainikainen, "The distribution of the product of independent Rayleigh random variables," IEEE Trans. Antennas Propag., vol. 54, no. 2, pp. 639-643, Feb. 2006.

[9] S. Ahmed, L.-L. Yang, and L. Hanzo, "Probability distributions of products of Rayleigh and Nakagami-m variables using Mellin transform," in Proc. IEEE ICC, Kyoto, Japan, Jun. 2011, pp. 1-5.

[10] M. Delibasic, M. Pejanovic-Djurisic, R. Prasad, "Performance analysis of dual-hop relay system over Ricean fading channels", Telfor Journal, Vol. 6, No. 2, 2014, pp. 92-96.

[11] D. Krstic, M. Stefanovic, R. Gerov, Z. Popovic, "Wireless relay system with two sections in $\kappa-\mu$ short term fading channel", The Twelfth Iinternational Conference on Wireless and Mobile Communications, ICWMC 2016, Nov. 13 - 17, 2016, Barcelona, Spain

[12] E. J. Leonardo and M. D. Yacoub, "Statistics of the product of arbitrary $\alpha-\mu$ variates with applications", 2014 IEEE 25th International Symposium on Personal, Indoor and Mobile Radio Communications, 2-5 Sept. 2014, Washington, DC, USA, pp. 73-76, DOI: 10.1109/PIMRC.20 14.7136135

[13] Z. Hadzi-Velkov, N. Zlatanov, and G. K. Karagiannidis, "Level crossing rate and average fade duration of the multihop Rayleigh fading channel", IEEE International Conference on Communications, 19-23 May 2008, Beijing, China, DOI: 10.1109/ICC.2008.835

[14] N. Zlatanov, Z. Hadzi-Velkov, and G. K. Karagiannidis, "Level crossing rate and average fade duration of the double Nakagami- $m$ random process and application in MIMO keyhole fading channels", IEEE Communications Letters, vol. 12, no. 11, Nov. 2008, pp. $822-824$, DOI: 10.1109/LCOMM.2008.081058

[15] G. K., Karagiannidis, N. C. Sagias, P. T. Mathiopoulos, "N*Nakagami: a novel stochastic model for cascaded fading channels," IEEE Transactions on Communications, Vol. 55, No. 8, 2007, pp. 1453-1458, doi:10.1109/tcomm.2007.902497

[16] X. Dong, N. C. Beaulieu, "Average level crossing rate and average fade duration of selection diversity", IEEE Communications Letters, 5(10), 2001, pp. 396-398, doi:10.1109/4234.957373

[17] M. D. Yacoub, J. E. V. Bautista, and L. G. de Rezende Guedes, "On higher order statistics of the Nakagami- $m$ distribution", IEEE Transactions on Vehicular Technology, Vol. 48, No. 3, May 1999, pp. 790-794.

[18] D. Aleksić, D. Krstić, Z. Popović, V. Milenković, M. Stefanović, "The analysis of wireless relay communication system in the presence of Nakagami- $m$ fading", WSEAS Transactions on Computer Research, Vol. 5, 2017, Art. \#7, pp. 51-62.

[19] D. Krstic, M. Stefanovic, P. Nikolic, S. Minic, „Statistics of the product of three Nakagami- $m$ random variables with applications", 26th International Conference on Software Telecommunications and Computer Networks 2018, Split - Supetar, Croatia, September 13-15, 2018, pp. 36-40, DOI: 10.23919/SOFTCOM.2018.8555819

[20] I. Vulic, D. Krstic, P. Nikolić, S. Minic, M. Stefanovic, “Average Fade Duration of Triple Nakagami- $m$ Random Process and Application in Wireless Relay Communication System", SpliTech2019 $4^{\text {th }}$ International Conference on Smart and Sustainable Technologies,
Bol/Split, Croatia, June18-21, 2019, doi:10.23919/splitech.2019.87830 94

[21] M. Nakagami, "The m-distribution: A general formula of intensity distribution of rapid fading", in W. C. Hoffman (ed.), Statistical Methods in Radio Wave Propagation: Proceedings of a Symposium held June 18-20, 1958. Pergamon Press, New York, pp. 3-36, 1960.

[22] Z. H. Yang and Y. M. Chu, "On approximating the modified Bessel function of the second kind", Journal of Inequalities and Applications, 2017(1): 41, doi: 10.1186/s13660-017-1317-z

[23] D. Ben Cheikh Battikh, "Outage probability formulas for cellular networks: contributions for MIMO, CoMP and time reversal features", Telecom Paris Tech. 2012. English

[24] K. Pearson, "Das Fehlergesetz und seine Verallgemeinerungen durch Fechner und Pearson. A Rejoinder" [The Error Law and its Generalizations by Fechner and Pearson. A Rejoinder], Biometrika, Volume 4, Issue 1-2, June 1905, pp. 169-212, doi:10.1093/biomet/4.1-2.169

[25] R. Priyadarshi, M. P. Singh, A. Bhardwaj, P. Sharma, "Amount of fading analysis for composite fading channel using holtzman approximation", 2017 Fourth International Conference on Image Information Processing (ICIIP). doi:10.1109/iciip.2017.8313759

[26] G. L. Stuber, Principles of Mobile Communications, Boston: Kluwer Academic Publishers, 1996.

[27] S. O. Rice, "Mathematical analysis of random noise", Bell Syst. Tech. J., vol. 23, July 1944, pp. 282-332.

[28] J. L. Lopez and P. J. Pagola, A simplification of the Laplace method for double integrals. Application to the second Appell function, Electronic Transactions on Numerical Analysis, vol. 30, 2008, pp. 224-236.

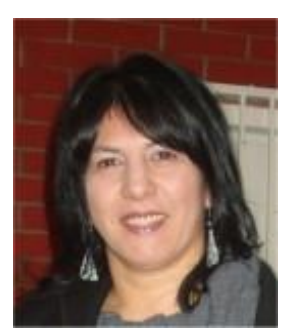

Dragana S. Krstic was born in Pirot, Serbia. She received the B.Sc, M.Sc. and $\mathrm{PhD}$. degrees in Electrical Engineering from Faculty of Electronic Engineering, University of Nis, Serbia in 1990, 1998. and 2006, respectively. She is working at Faculty of Electronic Engineering, University of Nis, since 1990. Her field of interest includes telecommunications theory, optical, wireless, mobile and satellite telecommunication systems. As author/co-author, she wrote about 260 scientific research papers, of which more than 60 are printed in international journals, several in national journals, 145 are referred at international symposia and conferences and around 35 at national. Dr Krstic had about 30 plenary and keynote lectures, panels and tutorials by invitation at international conferences and some faculties. She is also the member of editorial boards of a few international journals and reviewer for many. Also, she is a member of the technical program committees for near 120 conferences and reviewer for over new 130 ones.

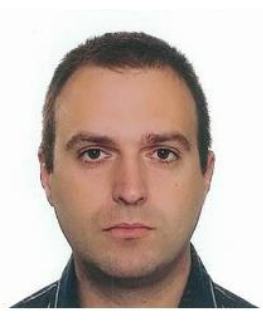

Petar B. Nikolic was born in Pirot, Serbia, in 1974. He graduated at the Faculty of Electronic Engineering, University of Nis, Serbia and received M.Sc. and Ph.D. degrees in 2008 and 2016, respectively. He is working in the company Tigar Tyres, Pirot. The main research interest is connected with the wireless communication systems. He has written or co-authored a considerable number of papers, published in eminent journals and conferences proceedings.

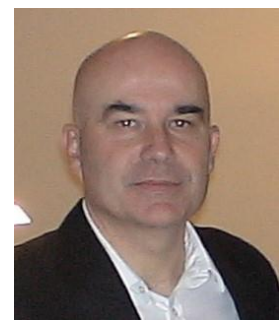

Ivan Vulic is Colonel and Assistant Professor at University of Defense, Belgrade, Serbia. Now he is vice-rector for scientific research at this University. 


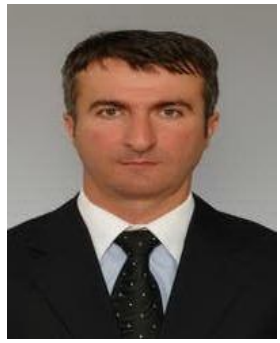

Sinisa G. Minic was born in Gulija, Leposavic Serbia. He received the B. Sc and M. Sc. degrees in electrical engineering from the Faculty of Electronic Engineering, in 1996 and 2001, respectively and $\mathrm{Ph}$. D. degree from Technical Faculty in Čačak, Serbia, in 2004. He is full professor at the Department of Informatics and Mathematics, Faculty of Teacher Education in Prizren - Leposavic University of Pristina Kosovska Mitrovica, Serbia, where he lectures: Computers in education, Information Technology, Educational technology, Informatics in pedagogy, Information technology in teaching. Areas of interest include telecommunications, information technology, information technology in education. Prof. Minic has authored over 50 scientific peer-reviewed papers and a large number of projects and patents. He is a member of the technical program committees and reviewer for several international journals and symposia.

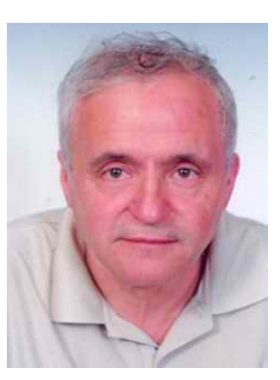

Mihajlo C. Stefanovic received the B. Sc., M. Sc. and $\mathrm{Ph}$. D. degrees in electrical engineering from the Faculty of Electronic Engineering, Department of Telecommunications, University of Nis, Serbia, in 1971, 1976 and 1979, respectively. His primary research interests are statistical communication theory, optical and wireless communications. He has written or coauthored a great number of journal publications. He has written five monographs, too. $\mathrm{He}$ was a mentor to hundreds of graduates, for dozens of master's theses and doctoral dissertations, and many times a member of commissions for the defense of such works. Now, Dr. Stefanovic is a retired professor at the Faculty of Electronic Engineering in Nis. 